\title{
PERIDYNAMIC MODELLING OF HARMONIC STRUCTURED MATERIALS UNDER HIGH STRAIN RATE DEFORMATION
}

\author{
THEODOR S. DE SOUSA ${ }^{1}$, AYLIN AHADI ${ }^{2}$, ELIS SJÖGREN ${ }^{3}$ AND DMYTRO \\ ORLOV $^{4}$ \\ ${ }^{1}$ Department of Mechanical Engineering \\ Box 118, S-22100 Lund \\ theodor.de sousa.889@student.lu.se \\ ${ }^{2}$ Department of Mechanical Engineering \\ Box 118, S-22100 Lund \\ aylin.ahadi@mek.lth.se \\ http://www.mecheng.lth.se/english/staff/aylin-ahadi/ \\ ${ }^{3}$ Department of Mechanical Engineering \\ Box 118, S-22100 Lund \\ elis.sjogren@material.lth.se \\ http://www.mecheng.lth.se/english/staff/show/elis-sj\%c3\%b6gren(6c45ff2b-9150-4d2e-b46b- \\ 20d4ec2a1334)/ \\ ${ }^{4}$ Department of Mechanical Engineering \\ Box 118, S-22100 Lund \\ dmytro.orlov@material.lth.se \\ http://www.mecheng.lth.se/english/staff/dmytro-orlov/
}

Key words: Peridynamics, Harmonic-structured materials, Peridigm

Summary. This paper applies the peridynamic continuum mechanics theory on a new type of material known as harmonic-structured materials. Using the Peridigm software, rapid uniaxial elongation is simulated on a peridynamic model of a thin bimodal harmonic-structured metal sheet. Mechanical wave initiation, propagation, and reflection, as well as fracture initiation and propagation are successfully simulated. 


\section{INTRODUCTION}

As society endeavours into new frontiers of possibility, the material industry and scientific community maintain pace by finding ways to improve the ductility, strength, deformation behaviour, weight, microstructures, etc. of materials. One of the methods for increasing strength is to decrease the grain size in the material, from coarse-grained (CG, $d_{C G} \geq 10 \mu \mathrm{m}$ ) [1] down to ultra-fine-grained (UFG, $d_{U F G} \leq$ $1.0 \mu \mathrm{m})[1]$, but this method also decreases ductility [2]. A possible solution proposed is to create a heterogeneous grain size distribution in a certain topology, creating what is denoted as a Harmonicstructured Material (HS) [1, 3-5]; The harmonic structure consists of evenly distributed cores of CG material inside a network of UFG material, as seen in Figure 1 on the following page. The grain sizes should differ at least by one order of magnitude, and the elastic behaviour and chemical composition must be the same for both UFG and CG materials.

A common tool to numerically investigate the plastic deformation behaviour of metals and alloys under mechanical loading is using finite element simulations [6-10]. Some recent efforts in modelling the behaviour of HS materials have also been carried out in e.g. [11-14]. However, FEM simulations based on classical continuum mechanics models are not able to resolve accurately phenomena at microscale at which the topology of harmonic structured materials originates. This increases the need to investigate whether continuum modelling with can be extended to include the modelling of discrete particles, and allow the explicit modelling of nonlocal phenomena, which inevitably influence the material behavior under loading. This approach is called peridynamics and is a novel non-local continuum model approach, which was developed in 2000 [15] is a non-local continuum mechanics theory based on an integral formulation of the equations of notion. Peridynamies has been successfulty used to model phenomena
at micro/nanoscale, see [16,17] and in this paper we employ peridynamics to sthulate the response of
thin bimodal harmonic-strictured metal sheet to high strain uniaxial load.
2 THEORY ON HARMONIC-STRU CTUREID MATERIALS
Strength is one of two key characteristics of materials [2]. It can be increased in a muttitude of ways, but is often correlated with a decrease of ductility [18], the other key characteristic of materials. One of

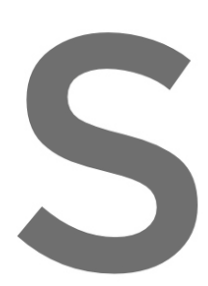

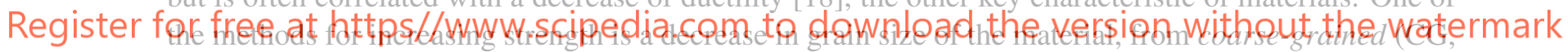

$d_{C G} \geq 10 \mu \mathrm{m}$ ) [1] down to ultra-fine-grained (UFG, $d_{U F G} \geq 1.0 \mu \mathrm{m}$ ) [1] microstructures, but this method

decreases the ductiitity significantily [2]. A proposed solution is to modify the structure heterogeneousiy, by featuring both CG and UFG microstructures in a bimodal fashion [19]. The maintained ductility originate from the coarse grains storing the lattice defects, while the UFG provide the increased strength [5].

[5] identifies an issue in common for the common methods of fabrication, namely topological distribution of coarse and fine grains. The topology of the materials is not easily reproduced and does not take full advantage of the respective grain size characteristics. For this issue, different topology controlling methods exist, whereof one is the method being the subject of this paper: Harmonic-Structured (HS) materials [3]. The structure consists of regularly distributed CG cores inside a UFG shell as seen in Figure 1 on the next page, both grain types possessing the same chemical characteristics.

The fabrication process to achieve the harmonic-structured material in a reproducible fashion is as follows [5]:

1. Creation of spherical powder particles using plasma rotating electrode processing [20] or similar methods. 


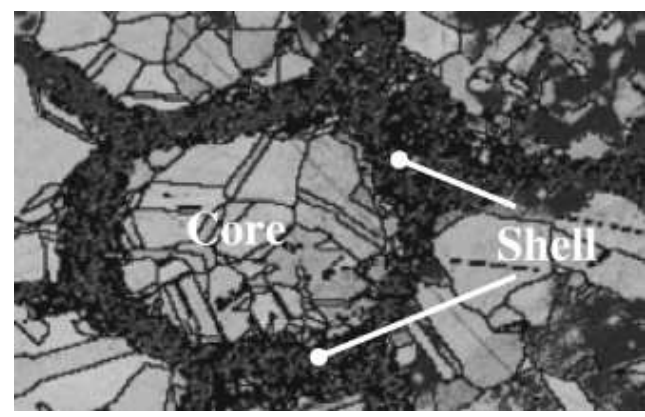

Figure 1: A conceptual illustration of the topology of the harmonic-structured material. A powder particle consisting of a CG core surrounded by a UFG shell that upon consolidation from a continuous UFG network. Image adopted from [14]

2. Cold working using mechanical milling or jet milling to decreases the grain size on the surface (often denoted as the shell) of the particles.

3. Consolidation of individual powder particles into full density specimens by using some pressureassisted sintering technique such as spark-plasma sintering, hot isostatic pressing, or hot-roll sintering, in vacuum to prevent formation of oxides.

A material type which shows an increase in strength and maintained ductility compared with its CG equivalent is harmonic structured nickel [1]. As seen in Figure 2, the ultimate stress point is elevated compared to both bimodal randomly distributed and CG nickel. In addition, the necking region is retained

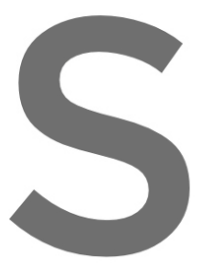
from the CG nickel unlike
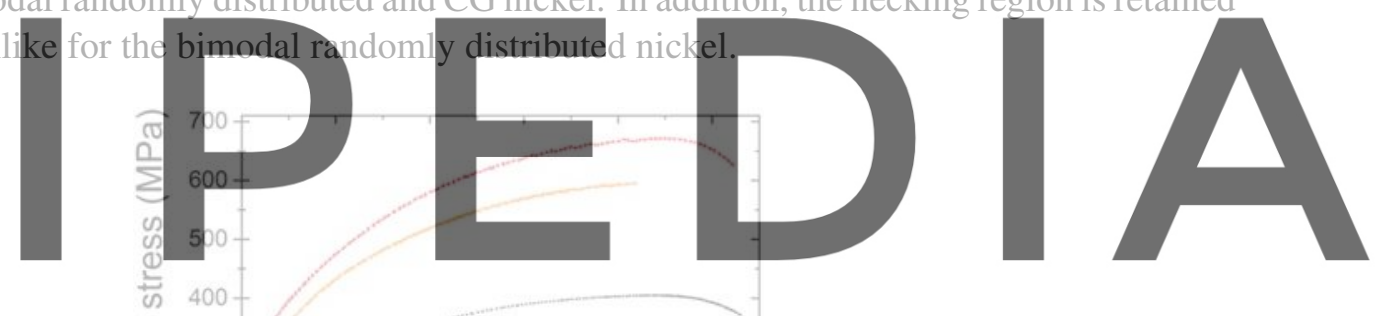

Register for free at https//www.sç्:pedia.com to dowrsload the version without the watermark

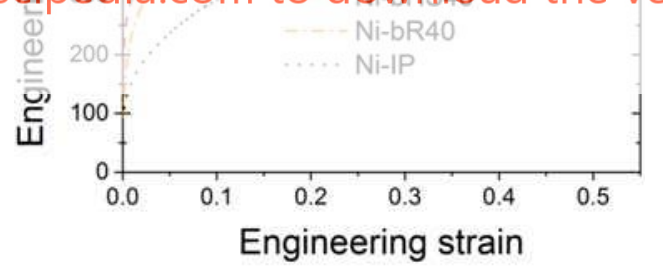

Figure 2: The stress strain curves measured in [1]. Ni-bHS40 and Ni-bR40 denotes the bimodal harmonic and random structured topology with a $40 \%$ fraction of UFG material. The Ni-IP is the corresponding CG nickel. Image adopted from [1].

The deformation process consists of an initial homogeneous elastic deformation (elastic properties of the UFG and CG materials in the HS material are the same), followed by a plasticity in the CG regions. This initial plasticity affects the stress strain-curve moderately, changing the slope inclination but maintaining a nearly linear behaviour during much of the stress increase, according to [5]. Knowledge of the behaviour following yielding in the CG region is as of yet not established fully, but is believed to 
be attributed to dislocation movement [1].

\section{THEORY ON PERIDYNAMICS}

Peridynamics, which was developed in 2000 [15], is a non-local continuum mechanics theory based on an integral formulation of the equations of motion. The equations of motion are valid even at discontinuities in the domain, making peridynamics suitable for fracture modelling [21]. The peridynamic domain, being a continuum theory, consists of an infinite amount of material points/particles $\mathbf{x}_{(k)}$, which are individually identified using their reference/initial coordinates $\mathbf{x}_{(k)}$. The material points possess a volume $V_{(k)}$, and a mass density $\rho\left(\mathbf{x}_{(k)}\right)$, as well as a strain energy density $W_{(k)}$. The points can be prescribed or subjected to body loads $\mathbf{b}_{(k)}\left(\mathbf{x}_{(k)}, t\right)$, displacements $\mathbf{u}_{(k)}\left(\mathbf{x}_{(k)}, t\right)$, or velocities $\dot{\mathbf{u}}_{(k)}\left(\mathbf{x}_{(k)}, t\right)$.

Three different peridynamic theories exists. The so-called bond-based peridynamics is the first of the three. It is cumbersome to use due to the lack of peridynamic formulations equivalent to classical continuum formulations, such as the Cauchy stress tensor. In order to address such issues, state-based peridynamics emerged [22]. The two state-based formulations are called ordinary and non-ordinary state based peridynamics. The ordinary state-based formulation allows peridynamic equivalents of CCM formulation, and the non-ordinary state-based formulation directly implements classical continuum descriptions of material behaviour.

Denote a material point by its initial coordinate $\mathbf{x}_{(k)}$, possessing a mass density $\rho_{(k)}$, as well as a strain energy density $W_{(k)}$. The point can be subjected to body loads $\mathbf{b}_{(k)}$, displacements $\mathbf{u}_{(k)}$, or velocities $\dot{\mathbf{u}}_{(k)}$. The material point interacts with all other points within a horizon $\delta$. The set of these points, the family

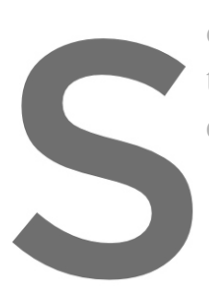
of $\mathbf{X}_{(k)}$, is denoted by $H$

to the force density state equation of motion for
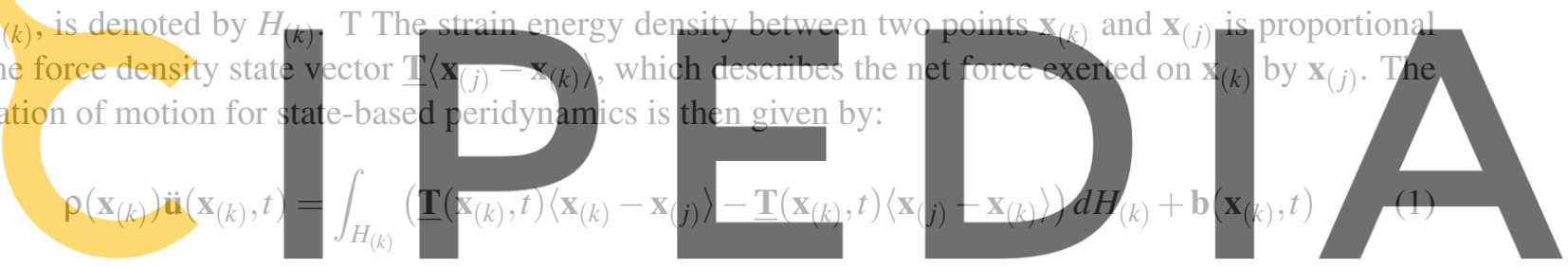

The state vector contains only the interactions of points having a bond with $\mathbf{x}_{(k)}$. When modelling

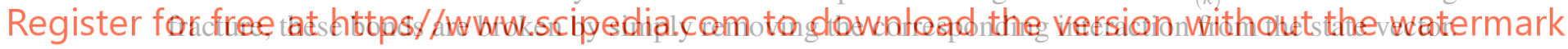
Bond breaking can be defined using a threshold, on the strain energy or the physical stretch, between two material points. These are commonly known as the critical energy, or critical stretch of the material [21].

Local damage ranges from 0 to 1 , where a 0 indicates that all bonds are intact, and a 1 is equivalent to all initial bonds being severed. Introducing the failure parameter $\mu$, and attributing the following boolean expression

$$
\mu=\left\{\begin{array}{l}
1 \text { if bond stretch below critical stretch } \\
0 \text { else }
\end{array}\right.
$$

allows the local damage to be formulated as

$$
\varphi\left(\mathbf{x}_{(k)}, t\right)=1-\frac{\int_{H_{(k)}} \mu\left(\mathbf{x}_{(j)}-\mathbf{x}_{(k)}, \mathbf{t}\right) d H_{(k)}}{\int_{H_{(k)}} d H_{(k)}}
$$

Boundary conditions are applied on volumes rather than surfaces, as is the case in classical continuum mechanics. Since peridynamics is a non-local theory, the constraints cannot simply be applied on surfaces. Instead a virtual boundary domain $R_{c}$ is introduced, onto which the constraint is applied. Its 
thickness should be the same as the horizon $\delta$ [23], to make sure that the true boundary fully experiences the value from that part of the family.

\section{METHOD}

The harmonic-structured domain was generated in MATLAB [24] using a matrix containing the coordinates, material block ID, and volume of every node, as explained in [25]. The domain is similar in design to [14], and consists of evenly spaced CG spheres surrounded by an UFG mesh, as seen in Figure 3. The far ends have been extended in order to contain the volumetric displacement, and are seen in turquoise and green. Denoting $\mathrm{x}$ as the length, $\mathrm{y}$ as the width, and $\mathrm{z}$ as the depth, the size of the domain measures $5.0 \mathrm{~mm}$ or 200 nodes in $\mathrm{x}, 1.0 \mathrm{~mm}$ or 40 nodes in $\mathrm{y}$, and a fifth of the width $(1.0 / 5=0.2) \mathrm{mm}$ or 8 nodes in z. The fraction of UFG nodes is $40 \%$, equivalent to the ratio found in both [14] and [1].

Figure 3: The discretised domain of the harmonic-structured material. The two blocks are illustrated in white (CG) and red (UFG). The colours of the end sides highlights two different node sets.

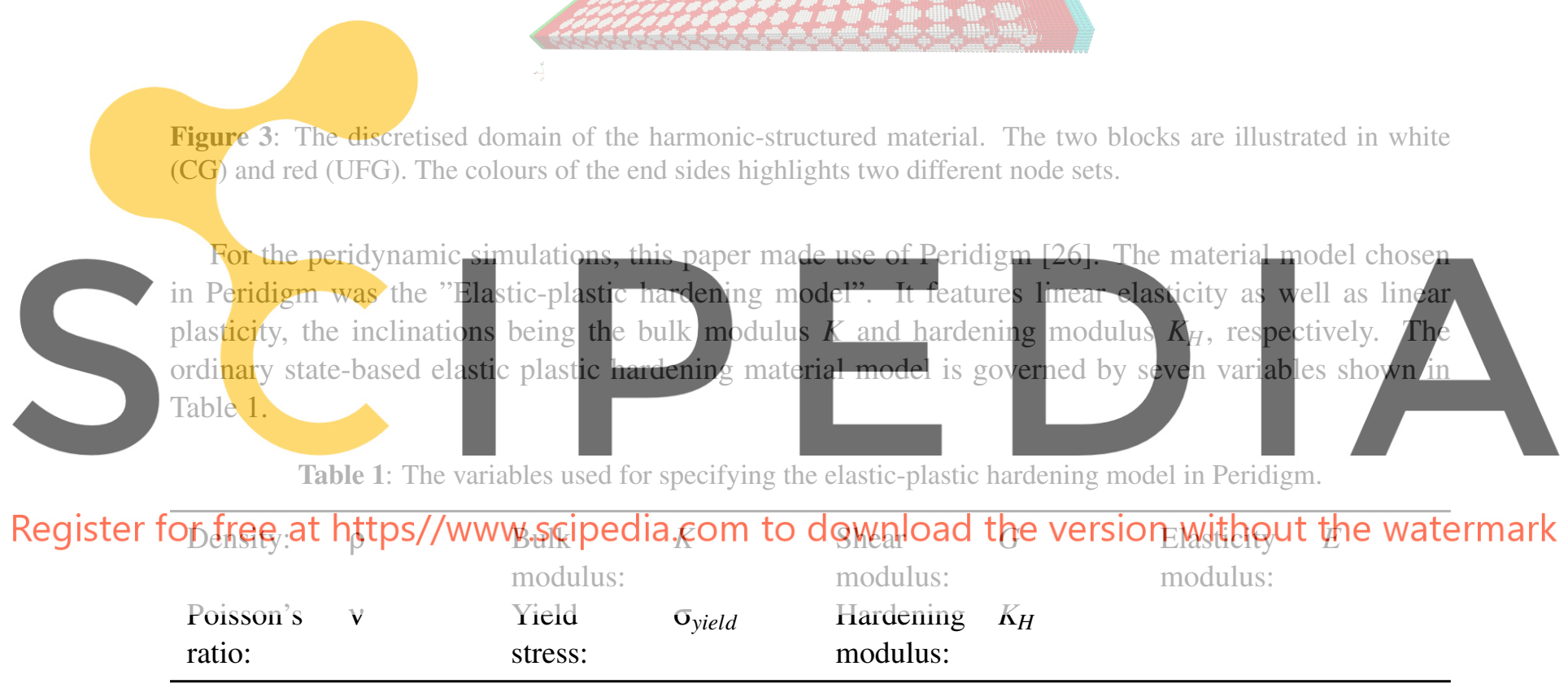

The internal code uses $K$ and $G$ for calculations, but engineering constants $(G, E, v)$ can be used instead as they are recalculated in Peridigm into $K$ and $G$. The grain material models both made use of the critical stretch damage model, with varying magnitude. In order to mimic the ductility of the CG material, and the more brittle behaviour of the UFG material, the stretch was arbitrarily chosen as 0.22 and 0.10 respectively, rather than calibrating the model with empirical data. The material and damage model parameters are shown in Table 2 on the next page. Simulations were made using the Velocity Verlet solver, described further in [25], with time steps $\delta t=1.78 \times 10^{-13} \mathrm{~s}$. The initial displacement rate of the ends of the domain were set to $\pm 225 \mathrm{~m} / \mathrm{s}$ initially, and in the second simulation $\pm 22.5 \mathrm{~m} / \mathrm{s}$.

The simulations were carried out on the Aurora computer cluster at LUNARC, the centre for scientific and technical computing at Lund University. The resulting data were post-processed in Paraview [27] for graphical presentation. 
Theodor S. de Sousa, Aylin Ahadi, Elis Sjögren, and Dmytro Orlov

Table 2: A summary of the material parameter values used in the paper.

\begin{tabular}{clllllll}
\hline Parameter: & Density: $\rho$ & $\begin{array}{l}\text { Elasticity } \\
\text { modulus: } \\
E\end{array}$ & $\begin{array}{l}\text { Poisson's } \\
\text { ratio: } v\end{array}$ & $\begin{array}{l}\text { Horizon: } \\
\delta\end{array}$ & $\begin{array}{l}\text { Yield } \\
\text { strength: } \\
\sigma_{\text {yield }}\end{array}$ & $\begin{array}{l}\text { Hardening } \\
\text { modulus: } \\
K_{H}\end{array}$ & $\begin{array}{l}\text { Critical } \\
\text { stretch: } s_{c}\end{array}$ \\
\hline UFG & 7850 & $210 \mathrm{GPa}$ & 0.31 & $\begin{array}{l}7.4 \times 10^{-5} \\
\mathrm{~m}\end{array}$ & $1502 \mathrm{MPa}$ & $4356 \times 10^{6}$ & 0.1 \\
$\mathrm{CG}$ & 7850 & $210 \mathrm{GPa}$ & 0.31 & $\begin{array}{l}7.4 \times 10^{-5} \\
\mathrm{~m}\end{array}$ & $185 \mathrm{MPa}$ & $898 \times 10^{6}$ & 0.22 \\
\hline
\end{tabular}

\section{RESULTS AND DISCUSSION}

The first simulation, with an initial displacement velocity of $2 \times 225=450 \mathrm{~m} / \mathrm{s}$ produces fracture when the initial shock wave from the displacement reaches the centre. The resulting shock wave from the rupture propagates back towards the end sides, reflecting off the boundary-condition nodes, causing further fracture in both end regions. The behaviour is illustrated using node damage in Figure 4 on the following page. Here, both non-damage nodes and fully damaged (free-floating) nodes are hidden. In Figure 6 on page 9 , the material type is displayed as red for UFG and white for CG.

Initially, the damaged zone expands inwards. At $t_{2}$ a $\Psi$-shaped region with higher damage manifests in the centre. No rupture occurs at this time, however, it can be seen in later time steps that fracture is initiated at the ends of the $\Psi$. The relatively low $s_{c}$ of the UFG material results in damage propagating

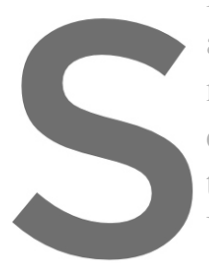
around the CG cores, nodes are hidden. Once th chunks of mass. As contacting the main domain. A close UFG material, with a core
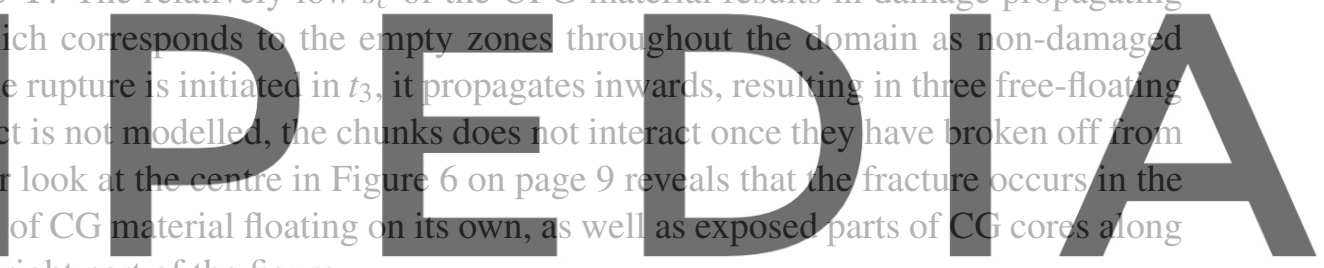

the fracture in the bottom right part of the figure.

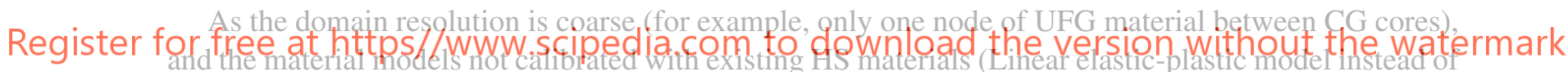

a power law model), the results cannot be used to say anything about the behaviour of real HS materials. Ideally, a three-dimensional domain would be utilised with a larger amount of UFG nodes separating the CG shells. Also, by applying the peridynamic theory, a continuum approximation is made. Given that the current theory on HS materials suggest that dislocation movement plays a central role in its behaviour, the continuum approximation might not be suitable at all. If that is the case, neither classical continuum mechanics simulations would be able to model the fracture behaviour. If the dislocation length scales are very small, the resource benefits of peridynamics over molecular dynamics might be lost since the number of nodes would need to increase in order to sufficiently model the dislocations.

The second simulation, with an initial displacement velocity of $45 \mathrm{~m} / \mathrm{s}$ did not produce a fracture, and shock wave propagation is illustrated using nodal velocity magnitude in Figure 5 on page 8. Velocity fluctuations can be seen before the centre is affected by the displacement. When the initial displacement shock waves pass each other, destructive interference is exhibited, with an area of effect expanding with the outward propagation of the shock waves. In the same manner as in the first simulation, the wave fronts reflect off the far ends of the domain, creating constructive interference in velocity magnitude as they propagate inward again. Any differences in magnitude between the UFG and CG materials are hard 

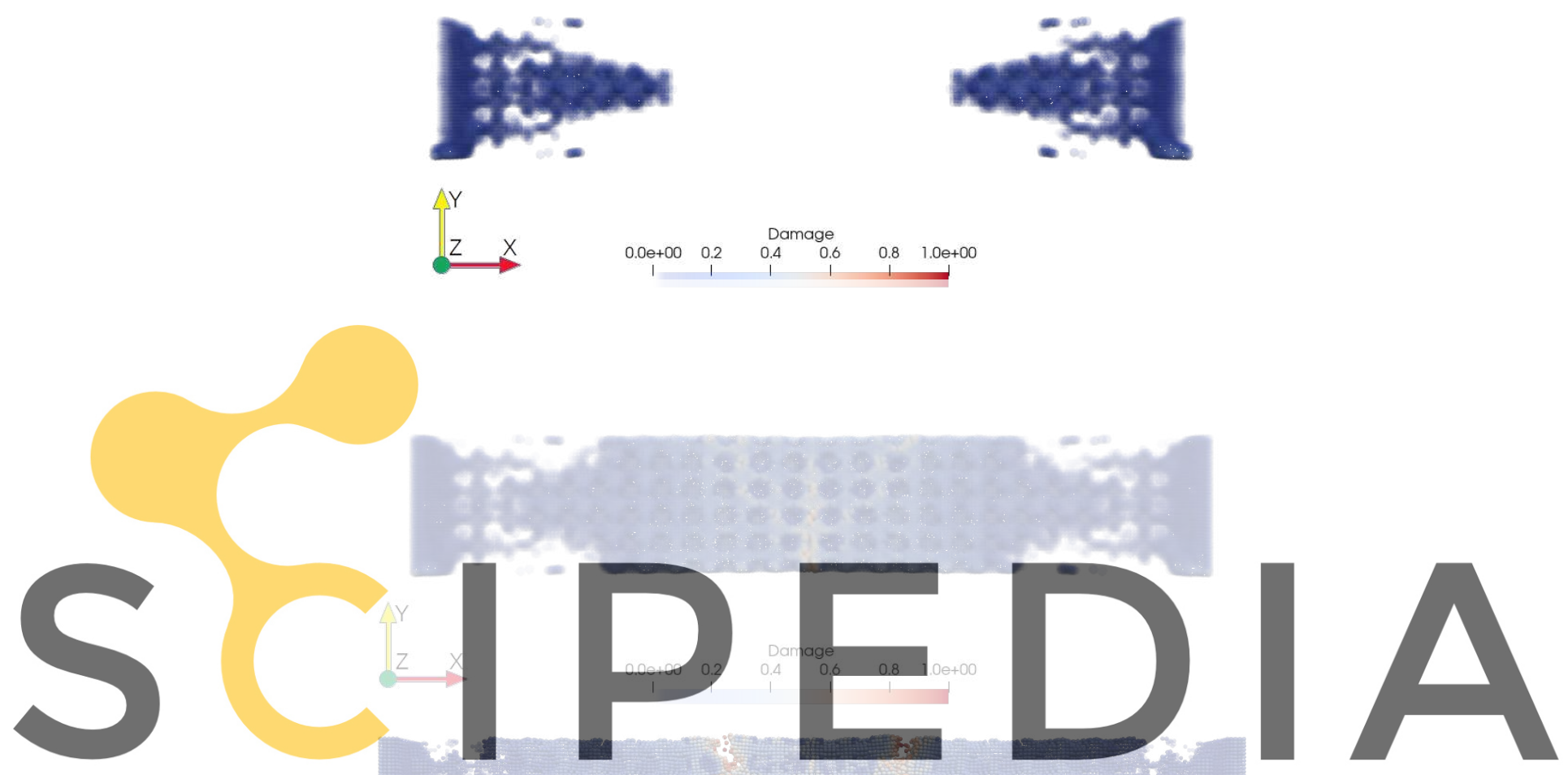

Register for free at https//www.scipedia.com to download the version without the watermark
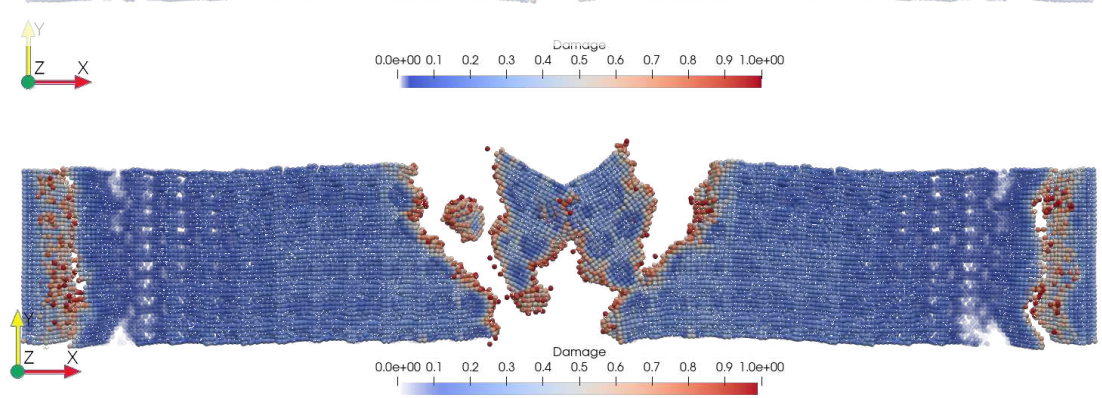

Figure 4: From top to bottom: Nodal damage at times $t_{1}=3.9 \times 10^{-7}, t_{2}=7.1 \times 10^{-7}, t_{3}=8.2 \times 10^{-7}$, and $t_{4}=11.0 \times 10^{-7}$ seconds. Nodal damage ranges from blue for low damage, to red for high damage. 


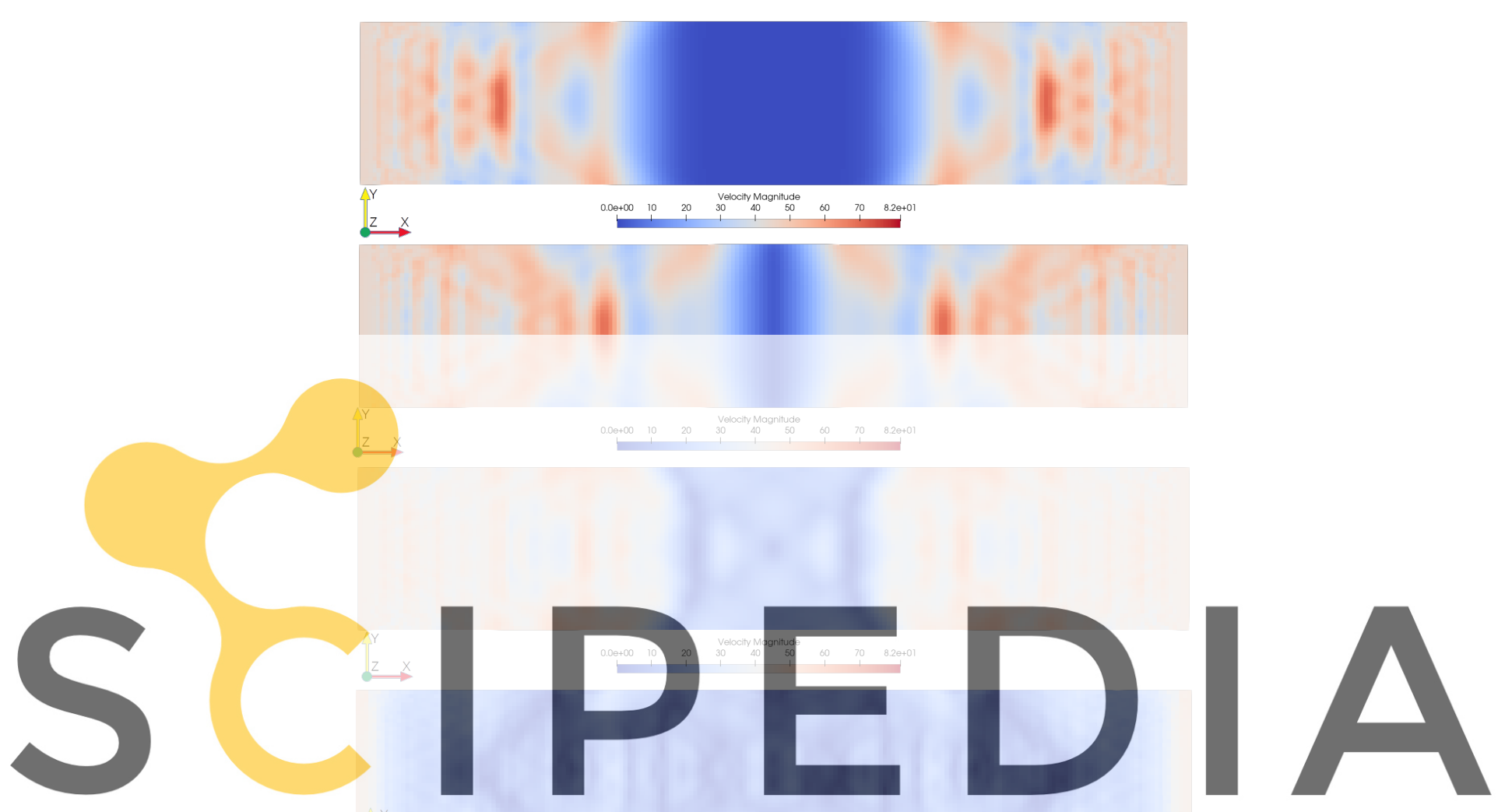

Register for free at https//www.scipedia.com to download the version without the watermark

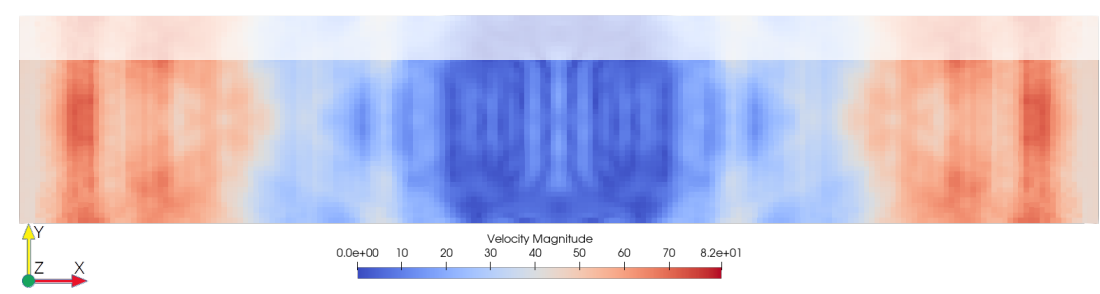

Figure 5: From top to bottom: Nodal velocity magnitude at times $t_{1}=2.9 \times 10^{-7}, t_{2}=4.0 \times 10^{-7}, t_{3}=5.6 \times 10^{-7}$, $t_{4}=8.8 \times 10^{-7}$, and $t_{5}=12.0 \times 10^{-7}$ seconds. Nodal velocity magnitude ranges from blue for low magnitude, to red for high magnitude. 


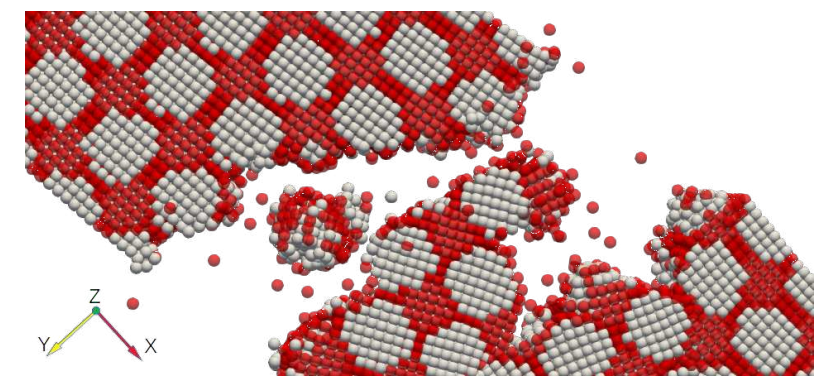

Figure 6: The centre fracture displayed with the two material types highlighted. UFG material displayed in red, and CG material in white. Time $t=10.3 \times 10^{-7}$ seconds

to distinguish, and would either way be distorted due to the coarse resolution of the domain.

The wave propagation is further noted to be symmetrical around the centre, both around the $\mathrm{X}$ axis and the $\mathrm{Y}$ axis. This is reasonable to expect since the nodes are positioned symmetrically and evenly spaced throughout the domain. Further work could preferably make use of a domain containing random perturbations of the nodes, in order to achieve more realistically modelled shock wave and fracture behaviour. Research could investigate fast paced dynamic fracturing, present in manufacturing processes such as milling or turning. Including contact modelling would further extend the research to cutting behaviour of the processes, taking advantage of the fracture modelling seen in the first simulation.

\section{CONCLUSION}

Fracture and shock

strain rate deformation

specimen fractures in the

ultra-fine-grained material couts

cuts through the UFG inate
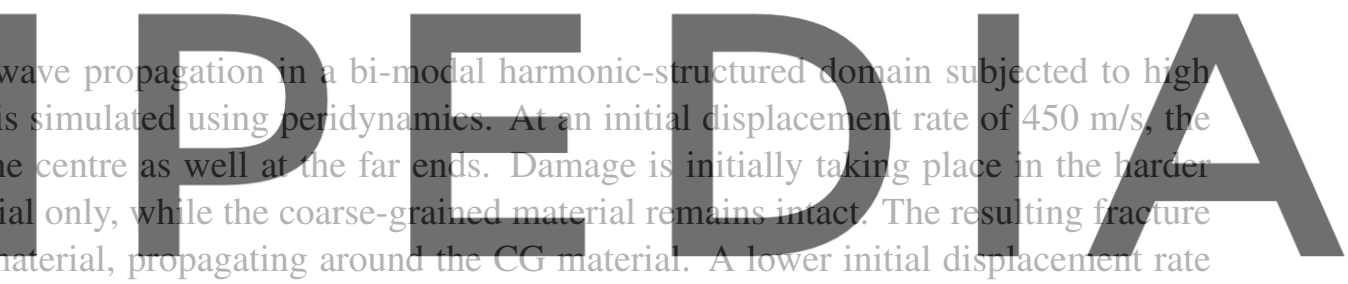

of $45 \mathrm{~m} / \mathrm{s}$ using the same material model does not produce fracture, but a clear wave interference be-

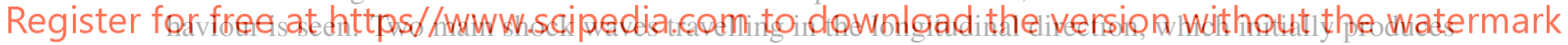

a destructive interference pattern, followed by a constructive interference pattern of the nodal velocity

magnitude once the shock waves recoil off the far ends of the domain.

The coarse resolution of the model domain, and the non-calibrated material model, prevent any conclusions from being drawn regarding the behaviour of real harmonic-structured materials. Further research should focus on refining the material model of the UFG and CG materials, as well as investigating how fracture propagation in the UFG shell and CG core is influenced by the domain resolution.

\section{ACKNOWLEDGEMENT}

All simulations were performed on the computer cluster Aurora on LUNARC, the centre for scientific and technical computing at Lund University. 


\section{REFERENCES}

[1] Dmytro Orlov et al. "Advantages of architectured harmonic structure in structural performance". In: IOP Conference Series: Materials Science and Engineering. Vol. 580. IOP Publishing. 2019, p. 012019.

[2] Yuri Estrin and Alexei Vinogradov. "Extreme grain refinement by severe plastic deformation: A wealth of challenging science". In: Acta materialia 61.3 (2013), pp. 782-817.

[3] Sanjay Kumar Vajpai et al. "Three-dimensionally gradient harmonic structure design: an integrated approach for high performance structural materials". In: Materials Research Letters 4.4 (2016), pp. 191-197. URL: https://doi.org/10.1080/21663831.2016.1218965.

[4] Dmitry Orlov, Hiroshi Fujiwara, and Kei Ameyama. "Obtaining copper with harmonic structure for the optimal balance of structure-performance relationship". English. In: Materials Transactions 54.9 (2013), pp. 1549-1553. ISSN: 0916-1821. DOI: 10.2320/matertrans . MH201320.

[5] Dmytro Orlov and Kei Ameyama. "Critical Assesment 37: Harmonic-structure materials - idea, status and perspectives". In: Materials Science and Technology 36.5 (2020), pp. 517-526. URL: https://doi.org/10.1080/02670836.2020.1719306.

[6] The Minerals Metals \& Materials Society (TMS). Modeling Across Scales: A Roadmapping Study for Connecting Materials Models and Simulations Across Length and Time Scales. Warrendale, PA: TMS, 2015, pp. xxiv, 93. ISBN: 9780692376065. DOI: 10 . 7449 / multiscale \_1. URL: www.tms.org/multiscalestudy.

[7] Franz Roters et al. "DAMASK-The Düsseldorf Advanced Material Simulation Kit for modeling
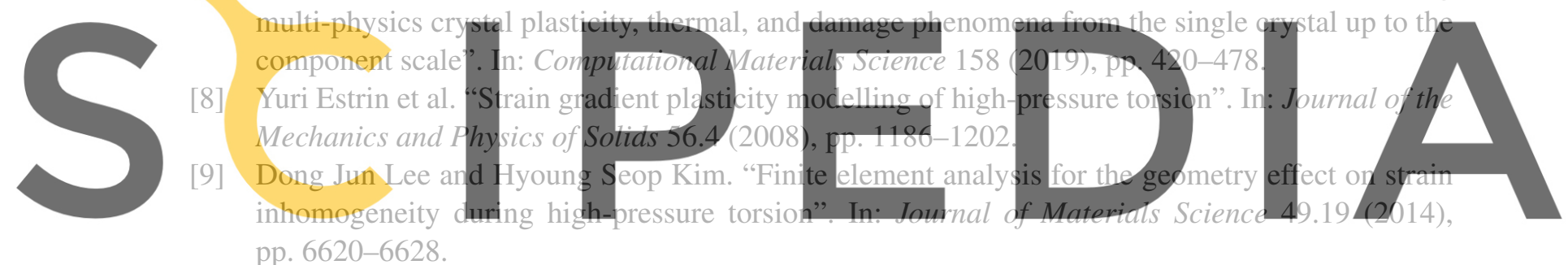
pp. 6620-6628.

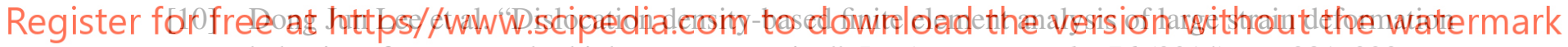
behavior of copper under high-pressure torsion". In: Acta Materialia 76 (2014), pp. 281-293.

[11] QD Ouyang, X Guo, and XQ Feng. "3D microstructure-based simulations of strength and ductility of bimodal nanostructured metals". In: Materials Science and Engineering: A 677 (2016), pp. 7688.

[12] Jia Liu et al. "A three-dimensional multi-scale polycrystalline plasticity model coupled with damage for pure Ti with harmonic structure design". In: International Journal of Plasticity 100 (2018), pp. 192-207.

[13] Xiang Wang et al. "A 3D crystal plasticity model of monotonic and cyclic simple shear deformation for commercial-purity polycrystalline Ti with a harmonic structure". In: Mechanics of Materials 128 (2019), pp. 117-128.

[14] Besim Ibishi. "Finite-Element Simulations of Harmonic Structured Materials". In: (2016). p. 40.

[15] Stewart A Silling. "Reformulation of elasticity theory for discontinuities and long-range forces". In: Journal of the Mechanics and Physics of Solids 48.1 (2000), pp. 175-209.

[16] Aylin Ahadi and Solveig Melin. "Capturing nanoscale effects by peridynamics". In: Mechanics of Advanced Materials and Structures 25.13 (2018), pp. 1115-1120. 
[17] Aylin Ahadi, Per Hansson, and Solveig Melin. "Indentation of thin copper film using molecular dynamics and peridynamics". In: Procedia Structural Integrity 2 (2016), pp. 1343-1350.

[18] William D Callister et al. Fundamentals of materials science and engineering. Vol. 471660817. Wiley London, 2000.

[19] Yinmin Wang et al. "High tensile ductility in a nanostructured metal". In: Nature 419.6910 (2002), pp. 912-915.

[20] NA Yefimov. Handbook of non-ferrous metal powders: technologies and applications. Elsevier, 2009.

[21] Erdogan Madenci and Erkan Oterkus. Peridynamics Theory and Its Applications. Springer, 2014. ISBN: 9781461484646. DOI: 10.1007/978-1-4614-8465-3.

[22] Stewart A. Silling et al. "Peridynamic States and Constitutive Modeling". In: Journal of Elasticity 88 (2007), pp. 151-184. DOI: 10.1007/s10659-007-9125-1.

[23] Richard W Macek and Stewart A Silling. "Peridynamics via finite element analysis". In: Finite Elements in Analysis and Design 43.15 (2007), pp. 1169-1178.

[24] MATLAB. 9.7.0.1319299(R2019b). Natick, Massachusetts: The MathWorks Inc., 2020.

[25] Martin Rädel. Peridigm Users Guide. Tech. rep. German Aerospace Center, 2018.

[26] Michael L Parks et al. "Peridigm Users' Guide v1. 0.0”. In: SAND Report 7800 (2012).

[27] James Ahrens, Berk Geveci, and Charles Law. "Paraview: An end-user tool for large data visualization". In: The visualization handbook 717 (2005). 